\title{
Status of salt interpretation for velocity model building in Brazilian basins
}

Fabien Marpeau* (CGG), Vincent Belz (CGG)

Copyright 2019, SBGf - Sociedade Brasileira de Geofísica

This paper was prepared for presentation during the $16^{\text {th }}$ International Congress of the Brazilian Geophysical Society held in Rio de Janeiro, Brazil, 19-22 August 2019.

Contents of this paper were reviewed by the Technical Committee of the $16^{\text {th }}$ International Congress of the Brazilian Geophysical Society and do not necessarily represent any position of the SBGf, its officers or members. Electronic reproduction or storage of any part of this paper for commercial purposes without the written consent of the Brazilian Geophysical Society is prohibited.

\section{Abstract}

For oil and gas exploration salt plays an important role. One of its main properties lies in its ability to deform under compressional environments and provide impervious anticlinal hydrocarbon traps. In Brazil, this phenomenon notably occurs at the base of salt itself and has been key to the recent so called "pre-salt" discoveries, such as in Santos basin. Yet, such rich tectonics give rise to very complex salt geometries. Allochthonous salt bodies are often found within the post salt sediments, with a much different velocity. This will cause velocity inversion algorithms to fail, unless salt is manually inserted into the model. For the past few years, great effort and resources have been allocated to salt interpretation as a main step of velocity model building, resulting in much improved structural imaging. In this paper, we review conventional approaches of top-down salt model building relying on human interpretation. Then, we analyze a more recent trend that consists of using Full wave-form Inversion strategies to mitigate salt uncertainties. We finally discuss a possible evolution of interpretation that would let machine learning reduce the human effort.

\section{Introduction}

In recent years, most of the largest Brazilian discoveries have been made in the pre-salt section (Figure 1a). This type of discovery may occur wherever the geological basement structural high goes shallow enough and forces the base of salt to deform. This results in anticlinal structures of the pre-salt sediments between the basement and base of salt. The base of salt provides an effective seal while the sides of the anticline convey desirable migration paths for hydrocarbons, see Figure 1b. A concern about salt is certainly the added complexity to the velocity model. The velocity of salt differs so much from the one of the surrounding sediments that current velocity inversion algorithms will diverge, unless salt is manually inserted into a separate layer of the velocity model. Typical velocity model building is currently carried out through so-called top-down approaches. First, the post-salt sediment velocity is derived from a sequence of velocity inversion iterations in which salt is overlooked. The resulting velocity field is considered correct above salt. Then, salt horizons are interpreted sequentially and salt velocity is artificially inserted into the velocity model. Finally, the pre-salt velocity is designed using various types of methods.

In Brazil, salt structures are mostly autochthonous, and until recently were considered simple and as such, given little attention. Salt interpretation was typically carried out by a separate contractor, implying large data transfers and significant bureaucracy. The resulting turnaround time between processing and interpretation was typically very long, yet still acceptable as long as only one pair of horizon was enough and no scenario was needed. However, the exploration of very deep water areas such as Campos, Santos and Espirito Santo basins has revealed very complex salt geometries, owing to their highly compressional environments. Salt interpretation requires significant effort, during the construction of the velocity model itself, and as such has become one of the main steps of velocity model building. Dedicated teams of interpreters have worked hand in hand with the processing teams to alternate velocity inversion and
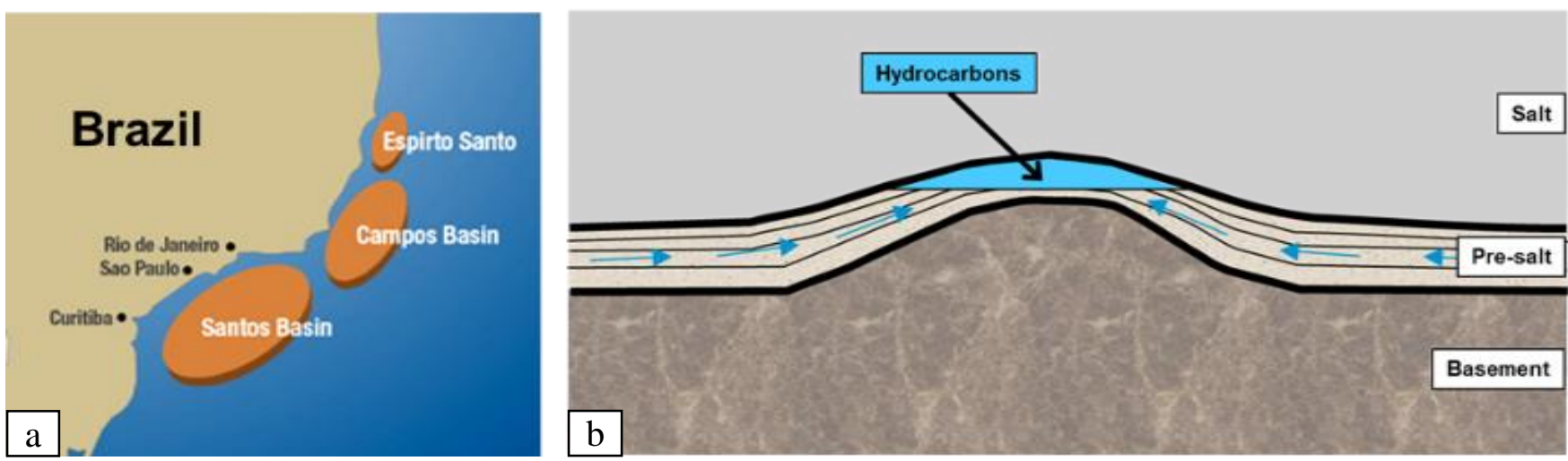

Figure 1: a) location of the main Brazilian salt structures; b) schematic example of pre-salt oil trap. The high of the base of salt is created by an elevation of the basement. The thining layer of carbonates in between provides a migration path for hydrocarbons to be trapped at the tip of the anticline. 


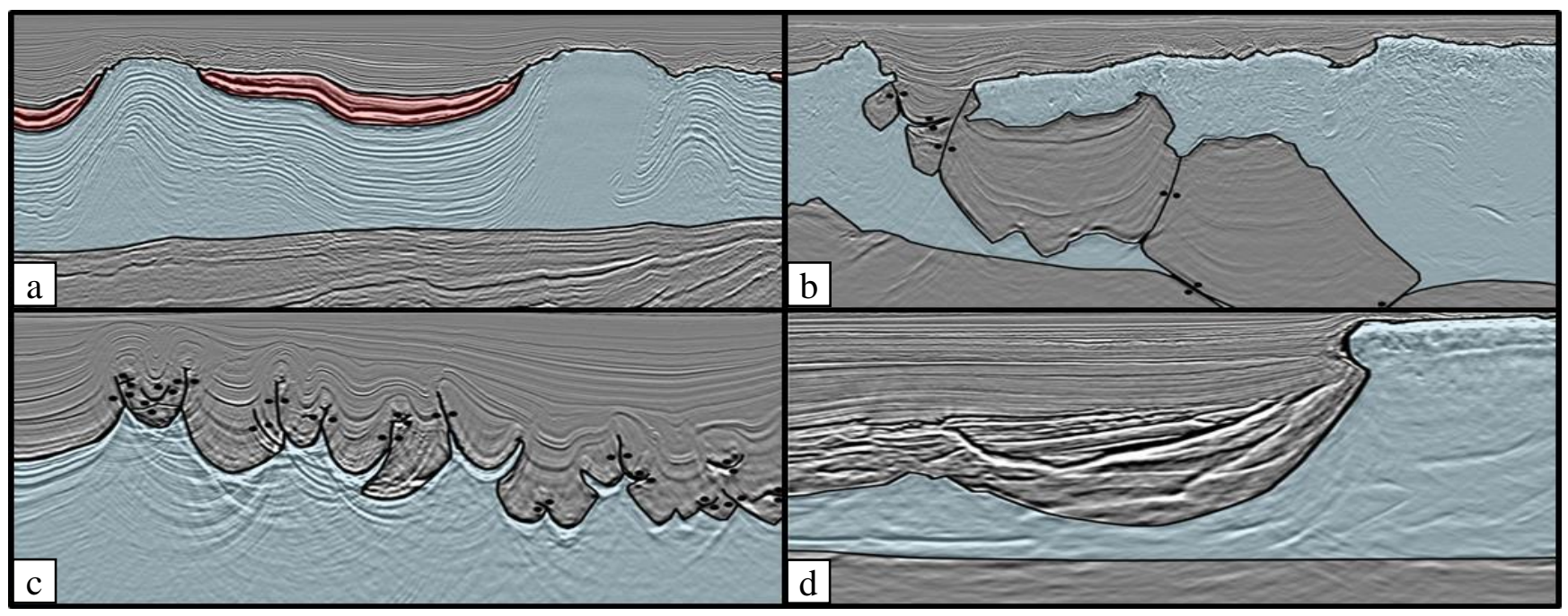

Figure 2: cross sections showing the complexity of the salt geometry in the very deep sater Santos basin; a) stratified autochtonous salt and tabular geometry; b) large overhang located in compressional area; overhang sub-salt exhibits several sediment packages separated by compressional welds; proper overhang sub-salt imaging is difficult but essential for correct pre-salt imaging; c) dense thrust fault system induces very complex ray-paths in the post-salt layer; observe the intra-salt migration noise; d) absorbing igneous intrusions in the post-salt and salt layers tend to hide the top of salt and base of salt events.

interpretation iterations back and forth with very quick turnaround. This has resulted in overall improved velocity models.

Yet, the top-down approach suffers from two limitations. First, it is a one dimensional concept: it assumes that the velocity at a given depth can be inferred only from vertically shallower seismic data. The other limitation is the fact that deeper information is not used to update the shallower layers. In practice, we remedy to these issues by obtaining a first velocity model through the top-down approach. Then, the resulting model is further improved by re-iterating a top-down approach until convergence, but allowing for deeper layers to be used to update the shallower layers. Such an iterative process can be very interpretation intensive, as salt horizons must also be updated every time the velocity above is updated.

Progress in FWI have promoted direct shaping of the salt, for example Zhang et al, (2018) and Michell et al. (2017). The updates given by FWI can also be used as a guide for new salt scenarios.

Recent advances of deep learning algorithms coupled with growing computational capacity have revolutionized the computer vision field. A notable example is provided by automatic image segmentation. Applying deep learning networks for the salt interpretation is one active area of research. The purpose is to replace the human intensive tasks and automate interpretation workflows. (Gramstad et al, 2018).

In the next section, we show examples of the complexity of salt interpretation for model building in Brazilian basins. Then, we present some examples for current trends which use FWI to guide interpretation. Finally, we focus on future developments involving machine learning algorithms to help reduce the labor intensive salt interpretation tasks.

\section{Salt scenarios to cope with complexity of Brazilian salt structures}

For the past few years, new explorations have exhibited complex salt structures in Brazilian basins. For instance, in Figure $2 \mathrm{a}$, salt is highly stratified into a continuous evaporitic sequence, topped with high velocity Albian carbonate layer. The position of the exact top of salt event is ambiguous and may be extrapolated from existing wells. In Figure $2 b$, large overhangs tend to hide the geology underneath. Some salt events, such as the secondary top, are invisible in practice and knowledge of salt tectonics is necessary to build the velocity model. Several horizon pairs are needed. In so called "top-down" approaches, each horizon is interpreted in a separate migration that places the desired event at its correct position. In Figure 2c, the presence of very complex thrust fault systems creates disturbances in the wave propagation. Salt was able to flow within hundreds of faults, resulting in very thin salt bodies with very fast velocity compared to the surrounding sediments. Another difficulty of the velocity model building is the presence of strong igneous activity observed in Brazilian basins. Volcanic rocks usually have a very distinct velocity and tend to absorb sonic energy, impairing the imaging of the underlying seismic events, see Figure $2 \mathrm{~d}$. While volcanic rocks in the post-salt section potentially make the interpretation of the top salt somewhat confusing, igneous intrusions at the bottom of salt hinder the interpretation of the base of salt itself, a key for hydrocarbon discoveries. The velocity model must be carefully evaluated in such situations. Velocity inversion algorithms, such as residual moveout tomography or full waveform inversion can be used to find the velocity of the igneous rocks in a similar fashion as the sediments. Another option is to interpret the top and base of volcanic rock packages so they can be treated separately. 


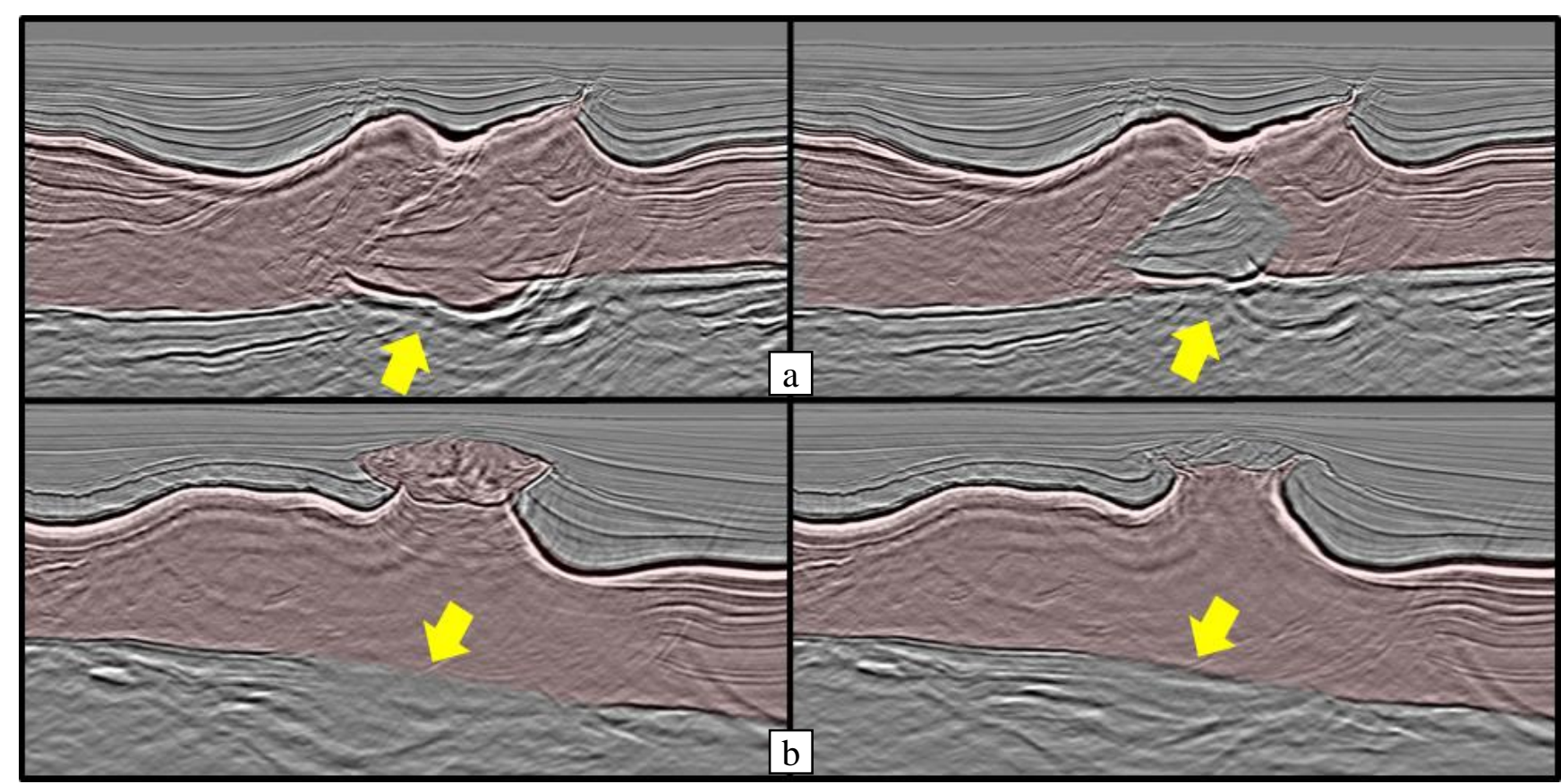

Figure 3: Scenario testing examples: a) on the left-hand side, the full salt model leads to a sag of the base of salt and partial destruction of the pre-salt carbonates; on the right-hand side, the encapsulated sediment model corrects for such an error; b) to the left, there was certainly too much salt in the velocity model, resulting in poorly visible pre-salt reservoirs. to the right, the new interpretation makes the base of salt and pre-salt sediments appear;

Such complexities gives rise to interpretation scenarios. Sediment encapsulation, a recurrent feature in the Santos basin, may be difficult to discriminate from salt stratifications in some instances. In Figure $3 a$, the interpretation of a stratified salt body as pure salt generates a spurious sag of the base of salt and deterioration of the pre-salt sediments. Looking more closely, a set of events appears dipping differently from the surroundings, with different seismic facies. It turns out that this set of events is a package of sediments encapsulated inside salt. After inserting sediment velocity, the base of salt is corrected and pre-salt sediments more continuous.

In Figure $3 b$, interpretation from previous processing mistook the top of a fault graben for the top of salt, resulting in too much salt in the velocity model. With new processing technologies imaging the graben better, it has become clear that the actual top of salt lies much deeper. The new, proper corresponding salt interpretation makes the base of salt and pre-salt carbonates appear in the image.

\section{Mitigating interpretation uncertainties with FWI}

Iterating between velocity inversion and salt interpretation through top-down velocity model building approaches has shown some limitations. First, every time the velocity is updated above salt, a significant amount of work is needed to update all the horizons underneath. Then, in some areas, the salt structures are so complex and/or difficult to visualize that too many scenarios may be necessary to have any hope to obtain the real solution. All this requires tremendous human effort in very localized areas. Even in areas where salt is clearly understood, inserting salt in the velocity model may sometimes be very ambiguous. This particularly occurs in numerous thrust fault systems, where the salt is so thin that the available frequency content does not allow for proper top and base discrimination.

Current velocity model building strategies lean towards a more approximate salt interpretation and letting computer algorithms cope with the refinement. Michell et al. (2017) and Shen et al. (2018) presented some recent examples of the successful application of full-waveform inversion to simultaneously update sediments and salt in the Gulf of Mexico using OBN data. A notable example in the Brazilian Santos Basin, with NATS data, is the use of time-lag full waveform inversion (TLFWI) (Zhang et al., 2018b) followed by image-domain reflection full waveform inversion (IRFWI). Starting from a complete and accurate salt model, the velocity is refined in the whole volume, including at the boundaries, and inside the salt itself. The advantage of using these FWI strategies is twofold: on the one hand, the time saving for interpreters is remarkable, when compared to a classical top-down strategy. Only a first, rather smooth interpretation is needed, possibly missing some details. The details of the salt need not be re-picked after the velocity inversion. On the other hand, FWI strategies may indicate major inaccuracies in the salt interpretation itself. For instance, significant velocity slowdown in large areas inside salt after FWI may indicate a sediment inclusion, or missed overhang. Such major changes in the velocity certainly calls for salt scenarios. However, contrary to purely interpretative salt scenarios such as the ones mentioned in the previous section, the foregoing scenarios are guided by the FWI 


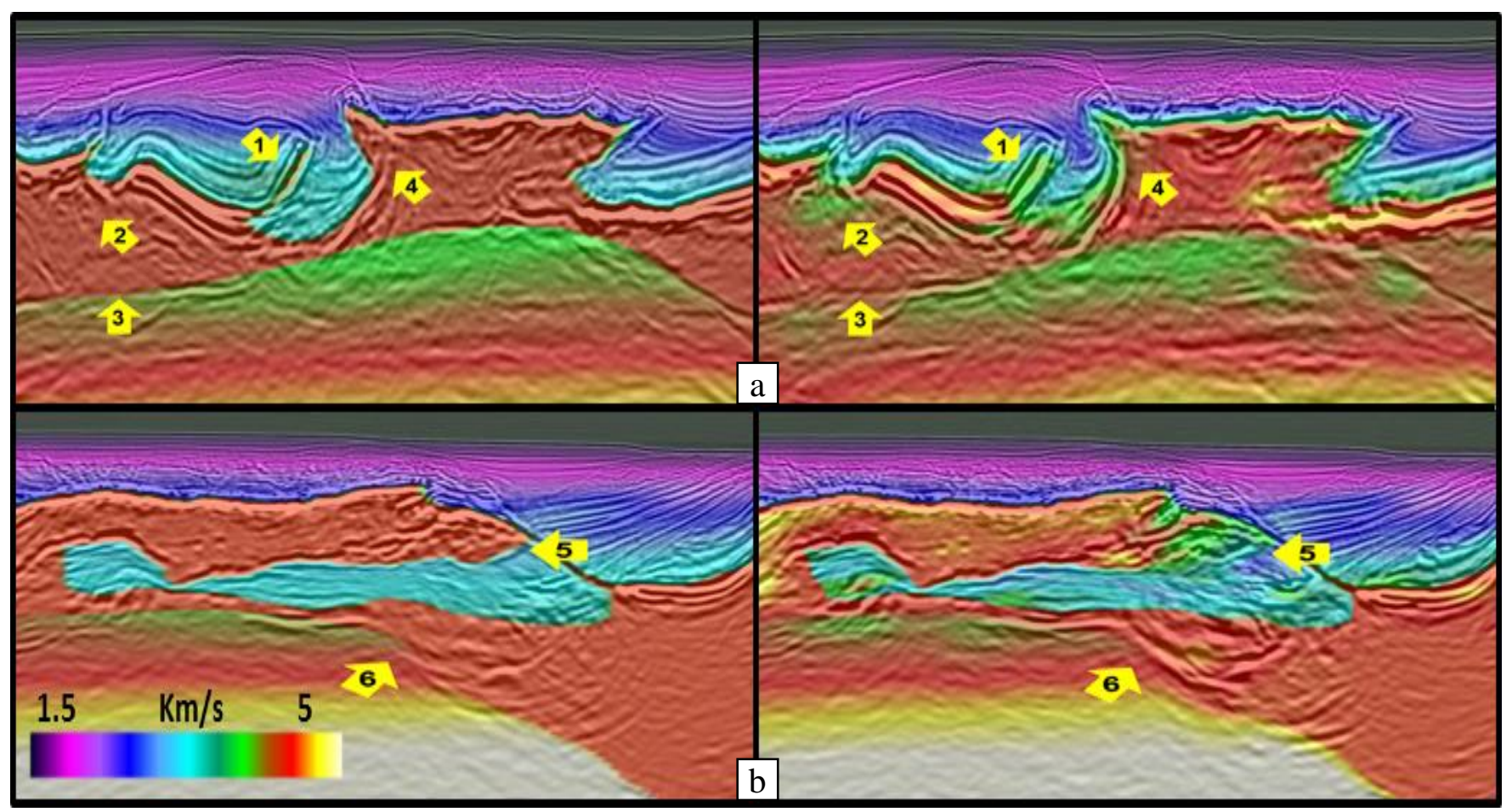

Figure 4: to the left, velocity model obtained through tomographic inversion, full waveform inversion and salt insertion; to the right, the same velocity model went through time lag and image based full waveform inversion; a) notice how the thrust fault velocity was updated in the new model (arrow 1), and a new overhang was carved (arrow 2); better velocity around the top of salt led to significant improvements of the imaging (arrows 3 and 4); b) the velocity update removed a piece of salt (arrow 5), allowing for better imaging of the structural high (arrow 6).

approach. This offers a lower risk potential test, ie: we modify the salt structures in areas where we already know that a change is required. In Figure $4 a$, we observe that a small salt overhang was carved through the FWI velocity update, without having to manually edit the salt model. The resulting image provide brighter base of salt and properly focused salt flanks. In Figure $4 \mathrm{~b}$, another example shows how subtle changes of the post-salt and intra-salt velocities obtained through TLFWI followed by IRFWI make the structural high appear in the image. It was not clearly visible before.

\section{Looking ahead: reducing labor intensive tasks through Machine learning?}

In most surveys, salt horizons are only partially visible. Using computerized horizon tracking, most of the top of salt horizon can be interpreted in a short amount of time, while it takes a considerable effort to manually interpret the area where automatic-tracking failed, usually where the top of salt reflectivity is low. Figure 5 a shows how computerized horizon tracking is able to design a preliminary top of salt horizon with little human input (interpreter defines some seeds that will be used as a guide through automatic tracking).

In an effort to decrease the required human effort, machine learning solutions have been the subject of intensive research. Initially, methods were mainly based on the computation of external attributes that would help the detection of salt in a supervised approach (Guillen, et al., 2015). More recently, Deep learning methods and in particular convolutional neural networks (CNNs) have been used for automated Salt interpretation (Waldeland, et al, 2018), (Gramstad, et al, 2018). CNNs outperform previous approaches with their capacity of automatically generating features that would best solve a particular classification task (Di et al, 2018). Additionally, The CNN classification is patch-based that incorporates local seismic reflection patterns for defining and learning the features.

Among the different architectures involving CNN, conditional adversarial networks have been effective to solve a wide variety of tasks for image processing including synthesizing photos from label maps, reconstructing objects from edge maps or colorizing image (Isola et al, 2017). These networks adapt well to new tasks with their capacity of learning a loss function rather than using an existing one.

Here we demonstrate what we believe is the current status of machine learning based interpretation. We focus only on the small task of top-of-salt interpretation. We use the pix2pix network (Isola et al, 2017). The network has been initially trained for Gulf of Mexico data (Zhang, et al., 2018a). Since Brazilian and Gulf of Mexico sections have different data distribution, we retrained the network with our data by using a transfer learning approach (Tan et al, 2018). 


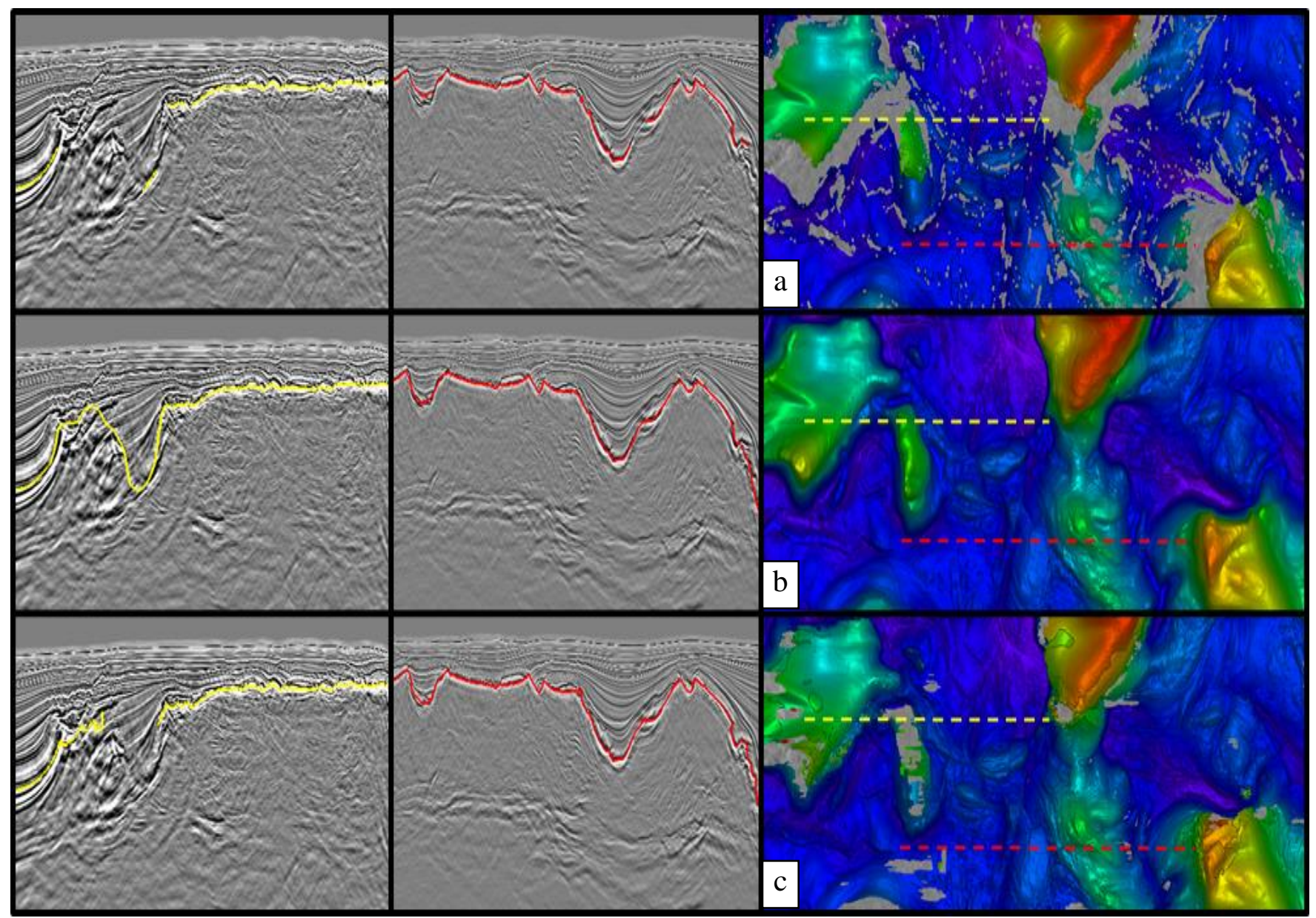

Figure 5: Top of salt interpretations; a) Computerized horizon tracking \& interpolation (b), Human horizon interpretation (c) Machine learning interpretation; The machine learning interpretation is very similar to the human interpretation. Yet, machine learning interpretation still underperforms in the areas that would require most of the human work, namely, the areas where the seismic event to be picked is uncertain.

Figure $5 \mathrm{c}$ shows the application of the network to interpret unseen data in Santos basin. We can see that the network is able to capture most of the simple structures of the top of salt. Still, the result is not perfect and some editions are required. Additionally, some areas lacking continuity are not perfectly captured by the network. However, these areas are typically the ones requiring most of the time of experienced interpreters, perhaps in the range of $95 \%$ of total interpretation effort. Figure $5 b$ shows a top of salt interpreted by an experienced interpreter. At the current stage, human interpretation is outperforming the CNN-based top of salt interpretation. Areas requiring more subjectivity (cf seismic section on the left part of figure 5) are more difficult to solve and would require further developments to improve the performance of the network. The value of machine learning is still not obvious. More research is required for these approaches to deliver impact.

\section{Conclusions}

Brazilian basins offer surprisingly complex salt geometries in very deep water areas. The integration of interpretation within the processing team has led to great improvement and opportunities for velocity model building in Brazilian surveys. It can be seen as a natural evolution from what has typically been done in Gulf of Mexico surveys. Short turnover between velocity inversion and salt interpretation allows us to run several tests and correct for errors continually during an imaging project, with no data transfers or bureaucracy. With a time scale of a few months, some imaging projects feature over a hundred salt scenario tests and a few thousands manual corrections. The same imaging project would likely take a few years to complete using contract interpretation.

To decrease the considerable interpretation work required in each project, a current approach is to let recently developed velocity inversion algorithms correct for salt interpretation inaccuracies while inserting proper, nonconstant salt velocity wherever needed. The improvement of the post-salt and intra-salt velocities results in better pre-salt imaging. This procedure requires a complete initial salt model, but no further manual updates is needed.

Future developments include machine learning algorithms, with the hope to further decrease the 
interpretation effort. Currently, CNN-based solution is not outperforming human interpretation. However, it can help experienced interpreters to focus on more subjective areas.

\section{Acknowledgments}

The authors would like to thank Florian Jouno (CGG) for some of the images presented herein, Daniela Donno and Adel Khalil for helping reviewing this article, and CGG Multi-client for the showrights to all images.

\section{References}

Gramstad, O., Nickel, M. (2018). Automated Top Salt Interpretation Using a Deep Convolutional Net: $80^{\text {th }}$ Conference \& Exhibition, EAGE, Extended abstract, TuD-09.

Waldeland, A. U., Jensen, A. C., Gelius, L., and Solberg, A. H. S. (2018). "Convolutional neural networks for automated seismic interpretation." The Leading Edge, $37(7), 529-537$.

Di, H., Wang, Z. and AIRegib, G. (2018) Why using CNN for seismic interpretation? An investigation. SEG Technical Program Expanded Abstracts 2018: pp. 2216-2220.

Guillen, P., Larrazabal, G., González, G., Boumber, D., \& Vilalta, R. (2015). Supervised learning to detec salt body. SEG Technical Program Expanded Abstracts, (pp. 18261829).

Isola, P., Zhu, J., Zhou, T. and Efros, A. A. (2017). Image-to-image translation with conditional adversarial networks: IEEE Conference on Computer Vision and Pattern Recognition (CVPR), 5967-5976.

Tan, C., Sun, F., Kong, T., Zhang, W., Yang, C. and Liu, C. (2018). A Survey on Deep Transfer Learning: 27th International Conference on Artificial Neural Networks, pp. 270-279.

Michell, S., Shen, X., Brenders, A., Dellinger, J., Ahmed, I., \& Fu, K. (2017). Automatic velocity model building with complex salt: Can computers finally do an interpreter's job. SEG International Exposition and 87th Annual Meeting, (pp. 5250-5254).

Shen, X., Ahmed, I., Brenders, A., Dellinger, J., Etgen, J., \& Michell, S. (2018, January). Full-waveform inversion: The next leap forward in subsalt imaging. The Leading Edge, pp. 67b1-67b6.

Zhang, Z., Long, L., Huang, K., \& Yu, D. (2018a). A machine learning approach for top of salt interpretation: Lessons learned. Workshop on Data Analytics and Machine Learning for Geoscience Applications. 88th SEG annual meeting and international exposition.

Zhang, Z., Mei, J., Lin, F., Huang, R., \& Wang, P. (2018b). Correcting for salt misinterpretation with full-waveform inversion. SEG International Exposition and 87th Annual Meeting, (pp. 1143-1147). 